\title{
Methadone and Torsade De Pointes in the Therapeutic Range
}

\section{Vannini MG, Soccorso P, Mazzone MR*, Piccioni A, Gubitosi G, Di Fazio F, Mandolini C, Pietropaolo L and Sighieri C}

Emergency Department Ospedale Vannini Rome Italy

${ }^{*}$ Corresponding author: Mazzone MR, Emergency Department Ospedale Vannini Rome Italy, E-mail: marinellamazzone@yahoo.it

Citation: Vannini MG, Soccorso P, Mazzone MR, Piccioni A, Gubitosi G, et al. (2014) Methadone and Torsade

De Pointes in the Therapeutic Range. J Clin Exp Res Cardiol 1(2): 201. doi: 10.15744/2394-6504.1.201

Received Date: November 03, 2014 Accepted Date: December 23, 2014 Published Date: December 27, 2014

\section{Introduction}

Methadone, a long-acting opioid agonist, binds to and occupies mu-opioid receptors, preventing withdrawal symptoms for 24 hours or longer, reduces craving for opioids, and, by maintaining high levels of opioid tolerance, reduces the euphoric effects of subsequent illicit opioid use. As a controlled substance with potential for abuse, methadone use is regulated in the US and other countries.

Methadone therapy is subject to a deep evaluation of the clinical case. The evaluation should include: urinary toxicological screening for heroin, cocaine, amphetamines, cannabinoids, benzodiazepines; full and deep medical history related to drugs, heart and liver disease and possible pregnancies for women; complete physical examination; blood and serological tests; cognitive and linguistic ability in a social context [1].

Some studies have noted a significant but weak relationship between the methadone dose and the degree of QTc prolongation at doses of up to $300 \mathrm{mg} /$ day. Methadone use, in both therapeutic doses and overdoses, has been associated with QTc interval prolongation and torsades de pointes, which in some cases has been fatal [2,3]. Many patients who develop torsades de pointes while taking methadone have other risk factors for this arrhythmia. Concern regarding the proarrhythmic potential of methadone prompted a physician safety alert from the US Food and Drug Administration (FDA). While the risk of QTc prolongation in methadone treated patients is controversial, it seems prudent to us to: inform patients of the potential risk of arrhythmia when they are prescribed methadone, ask patients about a history of structural heart disease, arrhythmia, or syncope [4].

Assess the patient for other risk factors for QTc prolongation [5].

\section{Case report}

A fifty-five-year-old man presented to our departement with relapsing syncope episodes. His past medical history included paranoid schizophrenia and chronic hepatitis HCV related.

Currently in methadone replacement therapy $280 \mathrm{mg} / \mathrm{die}$, quetiapine $300 \mathrm{mg} / \mathrm{die}$ and lorazepam 2,5 mg/die, due to his past history of drug of abuse related to heroin dependence.

The ECG showed polymorphic ventricular tachycardia run with torsades de pointes which resolved spontaneously and an episode of loss of consciousness requiring a synchronized DC shock 150J; long QT 440ms.

Laboratory tests revealed a mild hypokalemia (3,4 mEq/L). Mild hypokaliemia cannot cause torsade de point, just $\mathrm{K}<2.5 \mathrm{mEq} / \mathrm{L}$ is associated with arrhythmia. Hypokaliemia could be a risk factor. Association of drugs may be related with hypokaliemia.

Anyway it is treated with magnesium sulfate ( 2 gr bolus) and continuous intravenous infusion $4 \mathrm{mg} / \mathrm{min}$ and also for correction of hypokalemia; the administration of methadone was reduced.

Some days later the patient was asymptomatic and the haemodynamic parameters were normal with no arrhythmias.

The substance abuse treatment had to be reviewed by a specialist. In a following hospitalization due to pneumonia, the patient's ECG showed polymorphic ventricular tachycardia run, torsades de pointes, resolved spontaneously. Anamnesis revealed a persistent high ranking dosage of methadone and quetiapine treatment.

\section{Conclusion}

Sudden cardiac death has been known for decades to occur in association with taking some antipsychotic medications, especially in high dosage. We would like to stress that torsades de pointes can occur even with a range drug monitoring. 
There is no compelling research evidence to base a decision whether to obtain an ECG prior to the start of methadone treatment. On the basis of our clinical experience, an ECG is suggested in patients with cardiac risk factors, including a history of structural heart disease, arrhythmia, a family history of prolonged QTc, the patient having a history of a prolonged QTc, the patient being treated with other medications that can prolong the QTc, or syncope. Follow up ECGs in such cases should be obtained within 30 days of starting methadone and annually.

A reasonable strategy for the management of torsade de pointes caused by drug exposure is: correct hypoxia, hypokaliemia and hypomagnesemia even when aren't present; administer magnesium 1 to $2 \mathrm{~g}$ in $10 \mathrm{ml}$ in push even when the serum magnesium concentration is normal; consider lidocaine; consider electrical pacing overdrive at rates of 100min; consider pharmacologic overdrive pacing with isoproterenol at 2 to $10 \mathrm{mcg} / \mathrm{kg} \mathrm{min}$; supplemental potassium even when potassium concentration is normal. If the patient with Torsade de pointes is unstable use high energy unsynchronized shocks (defibrillation doses).

Most common medications associated with torsade de pointes are: sotalol, cisapride, amiodarone, erythromycin, quinidine, clarithtomycin, haloperidol, fluoxetine, digoxin, fluconazole, and furosemide.

Some patients have a sufficiently high risk of mortality or serious morbidity due to the opioid use disorder that the benefits of methadone outweigh the risks indicated by a prolonged QTC interval.

Potential risks and benefits of methadone therapy should be discussed with the patient for QTc interval $>450 \mathrm{msec}$ but $<500 \mathrm{msec}$, and the ECG should monitored. For QTc interval $>500 \mathrm{msec}$, consideration should be made for discontinuing or reducing the methadone dose; contributing factors (eg hypokalemia, other drugs) also should be eliminated and alternative therapy considered.

The serum potassium concentration reported like cause of torsade pointes is 3.5 to $5 \mathrm{mEq} / \mathrm{l}$ especially in patients taking digoxin. The patients with value of potassium like our patient often havent't symptoms.

Clinicians should be aware of interactions between methadone and other drugs that prolong the QTc interval, or that can slow methadone elimination.

Some research suggests that substitution of $(\mathrm{R}, \mathrm{S})$-methadone with $(\mathrm{R})$-methadone reduces the methadone-induced prolonged QTc interval among opioid-dependent patients. Further work is needed to confirm this finding.

\section{References}

1. Verster A, Buning E (2005) Buprenorphine: critical examination of the arguments.

2. Amato L, Davoli M, Mitrova Z (2011) Reviews and protocols published by the group drugs and alcohol Department of Epidemiology of the Regional Health Service, Lazio region Group editorial base of the Cochrane Drugs and Alcohol in collaboration with the Centre Smoking, Alcohol and Drug Institute of Health.

3. Replacement therapy in the case of opioid dependence (2013) Recommendations July 2013 revision of the Federal Office of Public Health (BAG) Swiss Society of Addiction Medicine (SSAM) of the Association of Swiss Cantonal Medical (AMCS)

4. Fasoli M and Furba P (2009) Indications and contraindications, clinical surveillance of maintenance treatment with methadone for opioid dependence in the light of new pharmacovigilance data.

5. ACLS for Experienced Providers Guidelines CPR ECC 2010 AHA.

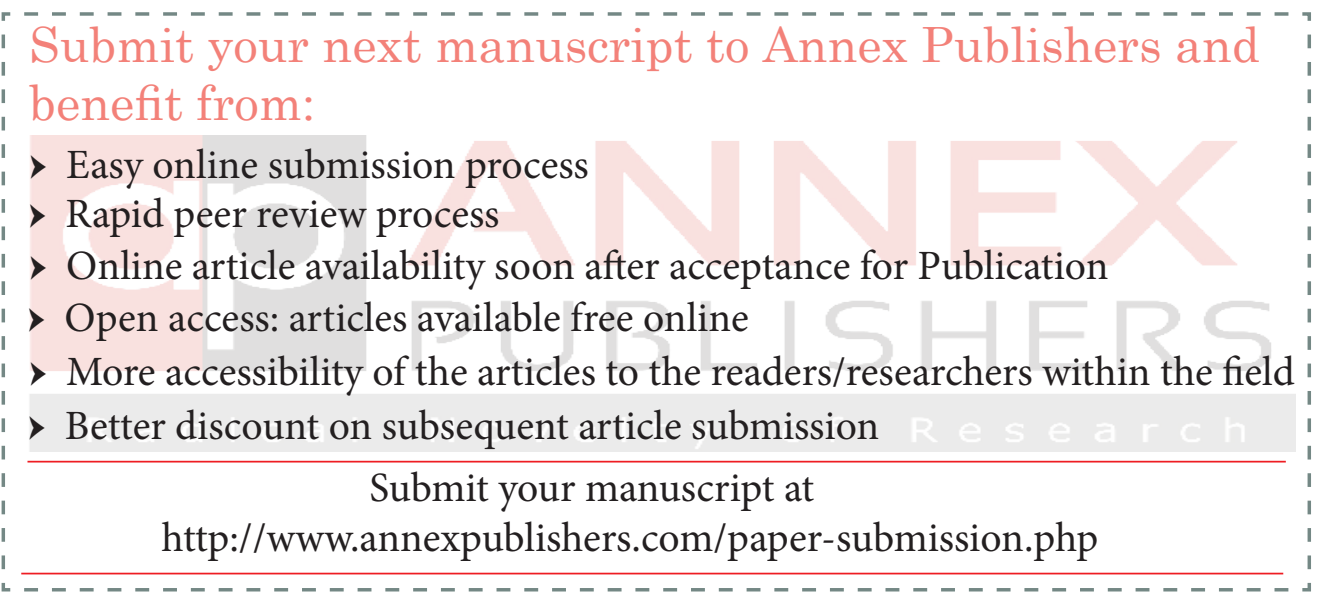

